\title{
Observation of ion gettering effects in high-temperature superconducting oxide material
}

\author{
S. H. Hong, M. L. Chen, J. Baniecki, and Q. Y. Ma \\ Department of Electrical Engineering, Columbia University, New York, New York 10027
}

H. A. Wang and R. W. Odom

Charles Evans \& Associates, Redwood City, California 94063

(Received 22 April 1996; accepted for publication 2 July 1996)

\begin{abstract}
Ion gettering effect has been observed in high-temperature superconducting $\mathrm{YBa}_{2} \mathrm{Cu}_{3} \mathrm{O}_{7}$ material. Silicon ions were implanted into the material and subsequent high-temperature annealing produced ion movement from a low concentration region to a higher concentration region where the damage of the crystal structure is severe. This gettering effect could be used to make a superconductor-nonsuperconductor-superconductor trilayer structure within a single YBCO film. (C) 1996 American Institute of Physics. [S0003-6951(96)04837-1]
\end{abstract}

In semiconductor technology gettering is a procedure where defects are intentionally produced at some distance from active layers, and unwanted mobile ion impurities move from the active space-charge region to this damaged area by a subsequent heat treatment. These intentional defects are preferentially on the back surface of the wafer or are spaced far away from the active region. Stable complexes can be formed with the vacancies or defects. ${ }^{1}$ Upon forming oxide precipitates $\left(\mathrm{SiO}_{x}\right.$, with $\left.x \sim 2\right)$, prismatic dislocations are generated in the Si matrix, which can 'getter' metallic impurities. $^{2}$ The use of ion implantation to produce the necessary defect density was described first by Buck et $a l^{3}$ and Seidel and Meek. ${ }^{4}$ Both groups used high-energy $\mathrm{He}^{+}$ion irradiation, ranging from 1.75 to $2 \mathrm{MeV}$, to produce iondamaged surface layers on silicon wafers and subsequent high-temperature annealing gettered unwanted electrically active impurities in silicon. Lecrosnier ${ }^{5}$ also reported gettering by ion implantation in III-V compounds and claimed that there are several different trapping regions inside materials after ion implantation.

In the high-temperature superconducting (HTS) oxide materials, such as $\mathrm{YBa}_{2} \mathrm{Cu}_{3} \mathrm{O}_{7}$, no reports on ion gettering effect have been presented. In this letter we present, for the first time, evidence for ion gettering in HTS oxide materials by ion implantation. We show that the gettered silicon ions form an insulating layer within the thickness of a YBCO film. Such a layer separates the surface layer and the layer near the substrate, creating a superconductornonsuperconductor-superconductor (SNS) trilayer structure.

The HTS films used were $c$-axis oriented YBCO films sputtered on $\mathrm{LaAl}_{2} \mathrm{O}_{3}(100)$ substrates. The films were typically $2000 \AA$ thick and had critical temperature $\left(T_{c}\right)$ about 85 $\mathrm{K}$ and critical current $\left(J_{c}\right)$ greater than $1 \times 10^{6} \mathrm{~A} / \mathrm{cm}^{2}$ at 77 $\mathrm{K}$. The YBCO films were implanted with silicon ions using an Eaton's 3206 ion implantor at an energy of $100 \mathrm{keV}$ and a dose of $1 \times 10^{16} / \mathrm{cm}^{2}$. After ion implantation, the YBCO films were annealed in oxygen at several different temperatures ranging from 650 to $1050{ }^{\circ} \mathrm{C}$ in $30 \mathrm{~min}$. The pressure in the furnace was maintained slightly above atmospheric pressure. The samples were characterized by secondary-ion mass spectroscopy (SIMS) depth profile for ion distribution and by susceptibility measurement for superconducting depth dependence.

Figure 1 shows SIMS depth profiles for silicon ionimplanted YBCO films with different post-annealing temperatures from 650 to $1050{ }^{\circ} \mathrm{C}$ using a quartz furnace with oxygen flowing. The SIMS analysis was performed with a PHI-6600 quadrupole mass spectrometer using cesium as the primary bombardment ion. Figure 1(a) is the silicon profile in the YBCO film after $650{ }^{\circ} \mathrm{C}$ annealing. The silicon ions are distributed over almost the entire film thickness with a peak at $0.1 \mu \mathrm{m}$ in depth. This profile is very similar to the one from an as-implanted film without annealing. As the

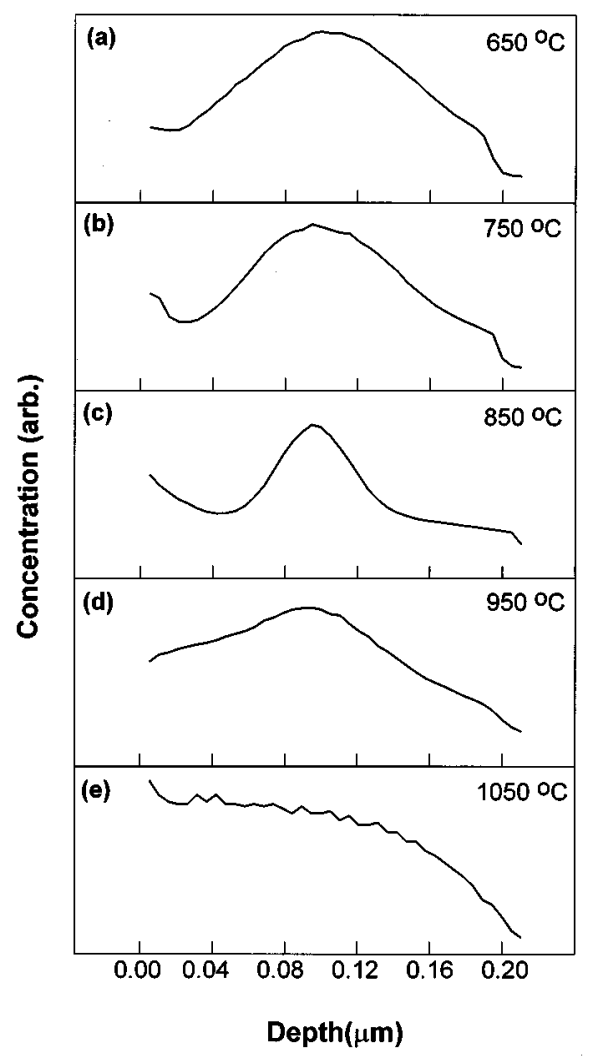

FIG. 1. Secondary-ion mass spectroscopy (SIMS) analysis for YBCO samples annealed at different temperatures. (a) $650{ }^{\circ} \mathrm{C}$, (b) $750{ }^{\circ} \mathrm{C}$, (c) $850{ }^{\circ} \mathrm{C}$, (d) $950^{\circ} \mathrm{C}$, (e) $1050^{\circ} \mathrm{C}$. 


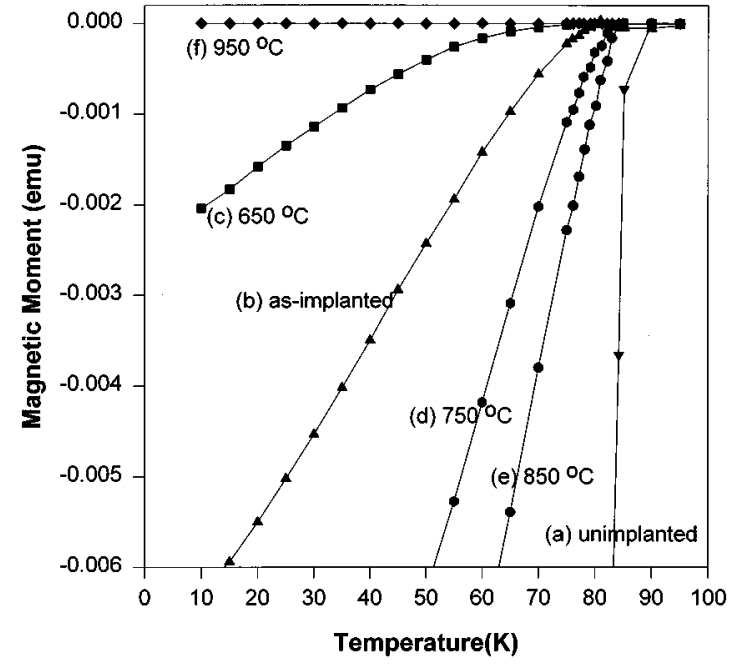

FIG. 2. Magnetic moment measurement of YBCO samples annealed at different temperatures. (a) unimplanted, (b) as-implanted, (c) $650{ }^{\circ} \mathrm{C}$, (d) $750{ }^{\circ} \mathrm{C}$, (e) $850{ }^{\circ} \mathrm{C}$, (f) $950{ }^{\circ} \mathrm{C}$.

annealing temperature increases, the ions move in an opposite direction of diffusion from low concentration region toward the peak concentration region. At an annealing temperature of $750{ }^{\circ} \mathrm{C}$ shown in Fig. 1(b), the silicon ions begin to getter towards the peak of the silicon concentration and the silicon profile narrows. The gettering process continues as the annealing temperature is increased and reaches the maximum gettering at $850{ }^{\circ} \mathrm{C}$ as in Fig. 1(c). At this temperature, the silicon ions are confined to a narrowband of film depth. The thickness of the band is about one-third of the film thickness. This band creates a SNS sandwich structure with two superconductor layers one near the surface and the other near the substrate. Further increases in the annealing temperature above $950{ }^{\circ} \mathrm{C}$ cause the ions to outdiffuse and intermix with the $\mathrm{YBCO}$ film. A uniformly intermixed silicon-YBCO film was obtained at $1050{ }^{\circ} \mathrm{C}$. This phenomenon can be explained in terms of a damage-related gettering effect. In the ion implantation process, the damage profile in the target is very similar to the implant depth profile which means that the crystal structure of the film is most severely damaged at the peak of the ion implant. The implanted ions lose most of their kinetic energy in this region and the nuclear stopping power cross section is maximum causing the most crystal damage. Above a certain threshold dose of implanted ions, the ions in less damaged area are attracted to the more damaged area of the films.

Figure 2 shows the susceptibility measurement of YBCO samples using a Quantum Design's magnetometer. (a) is a pure YBCO film which shows a critical temperature $\left(T_{c}\right)$ of $86 \mathrm{~K}$. (b) is the magnetic moment of as-implanted sample showing the suppressed $T_{c}$ of $72 \mathrm{~K}$. After $650{ }^{\circ} \mathrm{C}$ annealing in oxygen (c), the $T_{c}$ of the sample is further suppressed below $70 \mathrm{~K}$. This indicates that the region between implanted silicon ions and oxygen in the material is enhanced and silicon ions take more oxygen from the YBCO stochiometry to form a stable $\mathrm{SiO}_{x} \cdot{ }^{6}$ After $750{ }^{\circ} \mathrm{C}$ annealing (d), however, the magnetic moment curve moves back and $T_{c}$ increases to $81 \mathrm{~K}$. After further increase of annealing temperature to $850{ }^{\circ} \mathrm{C}(\mathrm{e})$, the $T_{c}$ reaches $82 \mathrm{~K}$. This regaining of diamagnetism indicates that superconductivity occurs in the layers above and below the ion gettered layer, as a result of ion getting effects as shown by SIMS data. After $950{ }^{\circ} \mathrm{C}$ annealing (f), superconductivity is completely gone.

Ion implantation has been one of the most powerful tools for impurity doping in semiconductor material. In HTS materials, ion implantation has been applied to modify the superconducting properties, ${ }^{6}$ damage the crystals of the materials, ${ }^{7}$ and to inhibit the conductivity. ${ }^{8,9}$ Clark et al. ${ }^{7}$ successfully used ion implantation in HTS thin films to alter the crystal properties of the YBCO. They used high-energy oxygen or arsenic beams in the energy range of $0.25-2.3$ $\mathrm{MeV}$ to physically damage the materials. In our previous work, ${ }^{8,9}$ we used reactive ions to inhibit the conductivity of the film and applied it to the device patterning. ${ }^{10}$ He et al. ${ }^{11}$ also used this reactive ion implantation (RII) method to make a pattern for their bolometer application.

One of the key issues for device patterning in HTS materials is developing useful multilayer structures since they can provide great flexibility in designing integrated high performance devices such as superconducting quantum interference devices (SQUID). The ion gettering effect observed in this study can lead to a trilayer superconductornonsuperconductor-superconductor (SNS) structure which may be utilized for multilayer HTS devices. Detailed study on the properties of these layers is in progress.

In conclusion, we have observed ion gettering effect in YBCO films after silicon implantation. The defect related gettering effect seems to be the main reason of this phenomenon. This effect results in the creation of SNS trilayer structure within a single film.

The authors would like to thank Dr. Yufei Yang and Michael Larkin and Professor G. M. Luke for their assistance. This work was partially supported by the National Science Foundation under Grant No. DMR-9531208.

${ }^{1}$ T. Y. Tan, E. E. Gardner, and W. K. Tice, Appl. Phys. Lett. 30, 175 (1977).

${ }^{2}$ W. K. Tice and T. Y. Tan, Appl. Phys. Lett. 28, 564 (1976).

${ }^{3}$ T. M. Buck, K. A. Pickar, J. M. Poate, and C.-M. Hsieh, Appl. Phys. Lett. 21, 485 (1972).

${ }^{4}$ T. E. Seidel and R. L. Meek, in Ion Implantation in Semiconductors and Other Materials, edited by B. L. Crowder (Plenum, New York, 1975), p. 305.

${ }^{5}$ D. Lecrosnier, Nucl. Instrum. Methods 209/210, 325 (1983).

${ }^{6}$ S. H. Hong, J. R. Miller, Q. Y. Ma, E. S. Yang, D. B. Fenner, C. Y. Yang, and J. I. Budnick, Mater. Res. Soc. Symp. Proc. 401, 309 (1996).

${ }^{7}$ G. J. Clark, A. D. Marwick, R. H. Koch, and R. B. Laibowitz, Appl. Phys. Lett. 51, 139 (1987).

${ }^{8}$ Q. Y. Ma, P. Dosanjh, J. F. Carolan, and W. N. Hardy, Appl. Phys. Lett. 63, 3633 (1993).

${ }^{9}$ S. H. Hong, J. R. Miller, Q. Y. Ma, E. S. Yang, and G. M. Luke, Appl. Phys. Lett. 67, 2717 (1995).

${ }^{10}$ Q. Y. Ma, A. Wong, P. Dosanjh, J. F. Carolan, and W. N. Hardy, Appl. Phys. Lett. 65, 240 (1994).

${ }^{11}$ X. He, J. Musolf, E. Waffenschmidt, K. Heime, H. Wolf, and K. Pierz, IEEE Trans. Appl. Supercond. 5, 2439 (1995). 